\title{
Synchronization of Chaotic Systems via Active Disturbance Rejection Control
}

\author{
Fayiz Abu Khadra \\ Mechanical Engineering Department, King Abdulaziz University, Rabigh, KSA \\ Email: fabukhadra@kau.edu.sa
}

How to cite this paper: Khadra, F.A. (2017) Synchronization of Chaotic Systems via Active Disturbance Rejection Control. Intelligent Control and Automation, 8, 8695.

https://doi.org/10.4236/ica.2017.82007

Received: March 7, 2017

Accepted: May 7, 2017

Published: May 10, 2017

Copyright $\odot 2017$ by author and Scientific Research Publishing Inc. This work is licensed under the Creative Commons Attribution International License (CC BY 4.0).

http://creativecommons.org/licenses/by/4.0/ (c) (i) Open Access

\begin{abstract}
This paper presents the use of active disturbance rejection control method (ADRC) to synchronize two different chaotic systems. The master system and slave systems have uncertainties and external disturbances. The numerical results are presented for the synchronization between the Duffing-Holmes system and the van der pol system. The numerical results presented show the effectiveness of the proposed method.
\end{abstract}

\section{Keywords}

Active Disturbance Rejection Control, Chaotic Systems, Synchronization

\section{Introduction}

The extreme sensitivity to initial conditions is a fundamental characteristic of a chaotic system. The response of a chaotic system depends largely on the initial conditions. A small change in the initial conditions can lead to big differences in the system states responses. A complete synchronization between two chaotic systems is defined as the act of forcing the states of a slave system to track exactly the states of a master system. Chaos synchronization has potential applications in secure communication and other applications.

A variety of approaches have been proposed and tested to achieve synchronization. Based on the Lyapunov method and linear matrix inequality (LMI) method, the adaptive synchronization of the Genesio-Tesi chaotic systems with three uncertain parameters was achieved in [1]. A new passivity-based synchronization method for a general class of chaotic systems was proposed in [2]. A control method, which uses an exact robust differentiator combined with a quasi-continuous high-order sliding mode-controller, was used in [3]. Adaptive feedback control design method, where controller parameters are assumed to be unknown and are evolved using adaptation laws so as to achieve synchronization 
was presented in [4]. The contraction theory for the adaptive synchronization was used in [5]. The adaptive synchronization and parameters identification of uncertain Rössler hyperchaotic system were presented in [6]. The use of active control to chaos synchronization between two different chaotic systems was reported in [7]. A robust indirect model reference fuzzy control scheme for control and the synchronization of chaotic nonlinear systems with uncertainties and external disturbances was proposed in [8]. The adaptive control method for synchronization of two Genesio-Tesi chaotic systems with uncertainties and dead-zone input was proposed in [9]. The synchronization of two Genesio-Tesi systems via single variable feedback controller was proposed in [10]. The synchronization between non-autonomous hyperchaotic systems viz., Liu and 4D hyperchaotic non-autonomous systems with parametric uncertainties using active control method was reported in [11]. The global asymptotical synchronization of chaotic Lur'e systems by using a delayed feedback proportional-derivative (PD) control scheme was reported in [12]. Based on a high order sliding-mode observer-identifier and a feedback state controller, the reducedorder synchronization problem of two chaotic systems with different dimension and relative degree was studied in [13]. In [14], a new surface sliding mode surface was proposed in an active sliding mode to synchronize two chaotic systems with parametric uncertainty. The synchronization of coupled unified chaotic systems via active control was presented in [15]. The direct adaptive interval type-2 fuzzy neural network (FNN) controller in which linguistic fuzzy control rules can be directly incorporated into the controller was developed to synchronize chaotic systems in [16]. Adaptive fuzzy logic controller (FLC) equipped with an adaptive algorithm to achieve $\mathrm{H} \infty$ synchronization performance for uncertain fractional order chaotic systems was reported in [17]. The chaos synchronization between Genesio chaotic systems with noise perturbation was reported in [18].

Most of the studies concerning chaos synchronization assume that all states of the systems are totally known and the parameters of the chaotic system are exact, which is not the case in practice. Therefore, a chaos synchronization study must consider the presence of uncertain parameters and external disturbances. One possible and efficient solution of the synchronization problem is to use an observer coupled with a controller. This methodology can be found in the Active Disturbance Rejection Control method (or ADRC) proposed in [19] and [20]. The ADRC method is based on the use of an extended observer coupled with a feedback controller in a closed loop control. The observer estimates all states of the system, the uncertainties and the external disturbances (total uncertainty). The total uncertainty is extended state of the system. If the estimation of the observer is accurate then the system to be controlled is converted to a simpler model, since total uncertainty is canceled in real time. In this way, a mathematical model of the system is not required.

In the present paper, the synchronization of different chaotic systems using the ADRC is presented. As an example, the synchronization of the Duffing- 
Holms and the van der Pol systems is considered to verify the proposed methodology.

\section{Synchronization Chaotic Systems}

A second order chaotic system such as the Duffing-Holmes (DHS) system and the van der Pol (VdP) systems can be written in the following general form

$$
\begin{aligned}
& \dot{x}_{1}(t)=x_{2}(t) \\
& \dot{x}_{2}(t)=f\left(t, x_{1}, x_{2}\right)+\Delta f\left(t, x_{1}, x_{2}\right)+\delta_{m}(t)
\end{aligned}
$$

$x_{1}(t), x_{2}(t)$ are the states of the master system, $f\left(t, x_{1}, x_{2}\right)$ is the nonlinear function of the master system $\Delta f\left(t, x_{1}, x_{2}\right)$ is the uncertainty and $\delta_{m}(t)$ is the external disturbance.

A salve system can be written as:

$$
\begin{aligned}
& y_{1}(t)=y_{2}(t) \\
& y_{2}(t)=g\left(t, y_{1}, y_{2}\right)+\Delta g\left(t, y_{1}, y_{2}\right)+\delta_{s}+u(t) .
\end{aligned}
$$

$y_{1}(t)$ and $y_{2}(t)$ are the states of the slave system, $g\left(x_{1}, x_{2}, t\right)$ is the nonlinear function of the slave system $\Delta g\left(x_{1}, x_{2}, t\right)$ is the uncertainty and $\delta_{s}(t)$ is the external disturbance, and $u(t)$ is the control input.

A totally unknown total disturbance can be defined as

$$
\sigma\left(y_{1}, y_{2}, t\right)=g\left(y_{1}, y_{2}, t\right)+\Delta g\left(y_{1}, y_{2}, t\right)+\delta_{s}(t) .
$$

The controlled slave system can be written as:

$$
\begin{aligned}
& y_{1}(t)=y_{2}(t) \\
& y_{2}(t)=\sigma\left(t, y_{1}, y_{2}\right)+u(t) .
\end{aligned}
$$

The unknown total disturbance $\sigma(x, y, t)$ is a bounded continuous and differentiable function with respect to time:

$$
\sigma(x, y, t) \leq D_{1} \dot{\sigma}(x, y, t) \leq D_{2} .
$$

The synchronization error between the slave system (2) and the master system (1) is defined as follows:

$$
e_{i}=y_{i}-x_{i} .
$$

A complete synchronization is to find a controller $u(t)$ so that the error states $e_{1}$ and $e_{2}$ converge to zero in finite time. In mathematical form

$$
\lim _{t-T}\|e(t)\|
$$

where $T$ is a finite and $\|\cdot\|$ is the Euclidean norm.

\section{ADRC Synchronization of Two Chaotic Systems}

The synchronization of two chaotic systems is considered as a tracking control problem. A tracking control problem can be formulated as: for the bounded states of the master system (1) design a controller $u\left(t, e_{1}, e_{2}, \sigma\right)$ that forces the states of the slave system $y_{1}$ and $y_{2}$ to track the states of the master system $x_{1}$ and $x_{2}$ in finite time. To solve this problem, the ADRC method is used as described below. 


\subsection{Extended States Observer}

To design a controller based on the measurement of the first state of the slave system, the errors $e_{1}, e_{2}$ and $\sigma$ must be available. One possible way to construct them is to use an extended observer (ESO) which is the main part of the ADRC. The main advantage of ESO is its ability to simultaneously estimate the total uncertainties online without the knowledge of an accurate mathematical model of the system. The ESO treats the total uncertainties as new state. An ESO for the slave system (4) is constructed as follows [20]:

$$
\begin{aligned}
& \dot{\hat{y}}_{1}=\hat{y}_{2}(t)-\alpha_{1} l_{1}\left(\hat{y}_{1}(t)-y_{1}(t)\right) \\
& \dot{\hat{y}}_{2}=\hat{y}_{3}-\alpha_{2} l_{2}\left(\hat{y}_{1}(t)-y_{1}(t)\right)+u \\
& \dot{\hat{y}}_{3}(t)=-\alpha_{3} l_{3}\left(\hat{y}_{1}(t)-y_{1}(t)\right) .
\end{aligned}
$$

The observer provides an estimate of the states of the slave system $\hat{y}_{1}, \hat{y}_{2}$. The third state $\hat{y}_{3}$ of the observer approximates $\sigma\left(t, y_{1}, y_{2}\right)$. Here, $l_{1}, l_{2}$ and $l_{3}$ are nonlinear function and $\alpha_{i}$ are tuning parameters. Using a constant high gain $\epsilon^{-1}$ with linear gain function the ESO can be written as:

$$
\begin{aligned}
& \dot{\hat{y}}_{1}=\hat{y}_{2}(t)+\frac{\alpha_{1}}{\varepsilon}\left(y_{1}(t)-\hat{y}_{1}(t)\right) \\
& \dot{\hat{y}}_{2}=\hat{y}_{3}+\frac{\alpha_{2}}{\varepsilon^{2}}\left(y_{1}(t)-\hat{y}_{1}(t)\right)+u \\
& \dot{\hat{y}}_{3}(t)=\frac{\alpha_{3}}{\varepsilon^{2}}\left(y_{1}(t)-\hat{y}_{1}(t)\right) .
\end{aligned}
$$

The tuning parameters $\alpha_{i}$ can be determined by using the pole placement method. Once the pole locations are chosen, the observer gains are obtained as follows [21].

The characteristic polynomial is calculated as:

$$
\lambda(s)=s^{2}+\alpha_{1} s^{2}+\alpha_{2} s+\alpha_{3} .
$$

Placing all the observers poles in the left half plane at $-\omega_{0}$ to make the characteristic polynomial Hurwitz.

$$
G(s)=\left(s+\omega_{0}\right)^{3} .
$$

Setting the above two equations equal and solving results in the following observer gains:

$$
\alpha=\left[\begin{array}{lll}
3 \omega_{0} & 3 \omega_{0}^{2} & \omega_{0}^{3}
\end{array}\right] .
$$

$\omega_{0}$ is called the observer bandwidth and it is the only tuning parameter. If the observer tuning procedure is adequate, the observer states converge to the system states $\hat{y}_{1} \rightarrow y_{1}, \quad \hat{y}_{2} \rightarrow y_{2}$, and $\hat{y}_{3} \rightarrow \hat{\sigma}$.

\subsection{Controller Design}

The control objective is to cancel the total disturbance and in the same time satisfying the tracking task. The ADRC controller is defined as follows:

$$
u=-\hat{y}_{3}+u_{0} \text {. }
$$


An example of such $u_{0}$ is a control signal from a feedback controller. Substituting (12) in (4) and assuming an accurate estimation of the total disturbance, the controlled slave system becomes:

$$
y_{2}=\sigma\left(t, y_{1}, y_{2}\right)-\hat{y}_{3}+u_{0} \approx u_{0} \text {. }
$$

The slave system is reduced to just a double integrator, which can be controlled with any classical controller design. If a linear proportional and derivative $(\mathrm{PD})$ controller is used, then the following control law can be obtained:

$$
u_{0}=k_{p}\left(x_{1}-\hat{y}_{1}\right)-k_{d}\left(x_{2}-\hat{y}_{2}\right)
$$

where $x_{1}, x_{2}$ are the states of the master system and $\hat{y}_{1}, \hat{y}_{2}$ are the estimated states of the slave system. One possible way to simplify the tuning of the controller is to set $k_{d}=2 \omega_{c}$ and $k_{p}=\omega_{c}^{2}$, where the $\omega_{c}$ is the closed-loop bandwidth of the controller. The methodology applied is represented in Figure 1.

\section{Numerical Simulation}

As an example, the synchronization of the DHS and the VdP chaotic systems is considered. The DHS system is the master system and VdP system is the slave system. The DHS equation describes the dynamics of nonlinear mechanical oscillator. This system has a cubic stiffness term to describe the hardening spring effect observed in many mechanical systems. The Duffing-Holmes is given by the following second order equation:

$$
m \ddot{x}+p_{0} \dot{x}+p_{1} x+p_{2} x^{3}=q \cos (\omega t)
$$

where $x$ is the oscillation displacement, $p_{0}$ is the damping constant, $p_{1}$ is the linear stiffness constant, $p_{2}$ is the cubic stiffness constant, $q$ is the excitation amplitude, and $\omega$ is excitation frequency. Equation (15) can be rewritten as twofirst order ordinary differential equations:

$$
\begin{aligned}
& \dot{x}_{1}=x_{2} \\
& \dot{x}_{2}=p_{0} x_{1}(t)-p_{1} x_{2}(t)-p_{2} x_{1}^{3}(t)+q \cos (\omega t) .
\end{aligned}
$$

To show the behavior of uncontrolled DHS, the following values of parameters are assumed: $\quad p_{0}=1, p_{1}=0.25, p_{2}=1, q=0.3$ and $\omega=1.2 \mathrm{rad} / \mathrm{sec}$.

Figure 2 shows the response of DHS system and the phase plot for the initial conditions

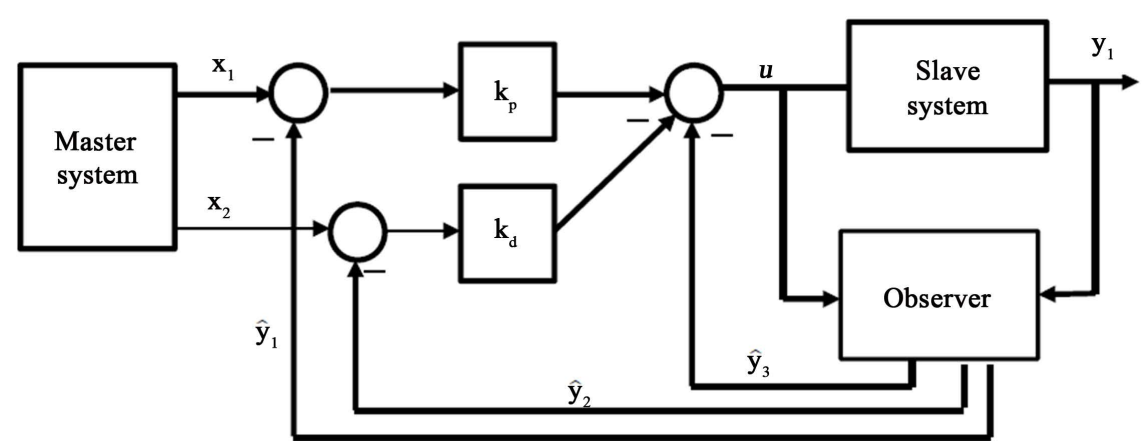

Figure 1. Closed loop control scheme for a master-slave synchronization using ADRC. 


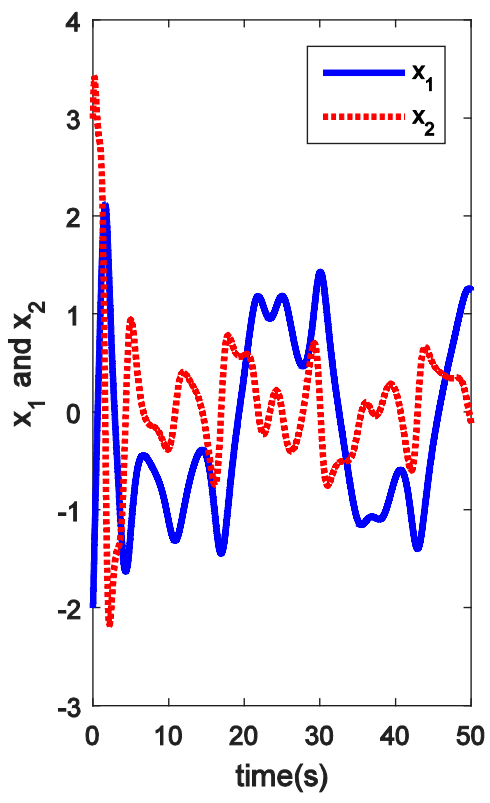

(a)

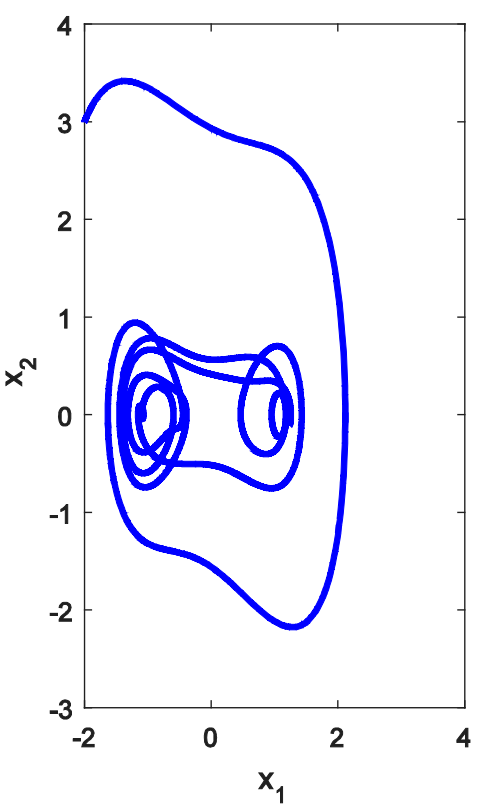

(b)

Figure 2. (a) The response of uncontrolled DHS; (b) The phase plot.

$$
x_{10}=-2, x_{20}=3 \text {. }
$$

The slave system is the Van der Pol system.

$$
\ddot{y}-\mu\left(1-y^{2}\right) \dot{y}+y=2 \sin (0.1 \pi t)
$$

where $\mu>0$ is a scalar parameter. Equation (17) can be rewritten as two-first order ordinary differential equations:

$$
\begin{aligned}
& \dot{y}_{1}=y_{2} \\
& \dot{y}_{2}=-2 y_{1}+3\left(1-y_{1}^{2}\right) y_{2}+2 \sin (0.1 \pi t) .
\end{aligned}
$$

To show the behavior of uncontrolled vdp system, its response to the initial conditions $x_{10}=0.8$ and $x_{20}=0.5$ is depicted in Figure 3 .

The chaos synchronization based on the methodology described in Section 3 is presented. Simulations are run for 50 seconds with a step size of 0.01 . The initial conditions for the two systems are the same as in the uncontrolled cases.

The following bounded external disturbance $\delta_{m}(t)$ is added to the master system:

$$
\delta_{m}(t)=0.1 \sin (t)
$$

To the slave system a bounded uncertainty $\Delta f\left(y_{1}, y_{2}, t\right)$ for simulation purposes is added as:

$$
\Delta f\left(y_{1}, y_{2}, t\right)=0.1 \sin (t) \sqrt{y_{1}^{2}+y_{2}^{2}}
$$

and the external disturbance is given by:

$$
\delta_{s}(t)=2 \sin (0.1 \pi t)+3 \sin (0.2 \sqrt{t+1}) .
$$

The controller parameter is $\omega_{c}=10$. The extended observer parameter is selected as $\omega_{0}=200$. 
From Figure 4 and Figure 5, it is clear that the states of the slave system completely track the states of the master system in very short time. Figure 6 shows the errors $e_{1}$ and $e_{2}$ as function of time, which shows that the synchronization error is tending to zero. It means that the slave systems can be synchronized successfully to the master systems. The control signal is shown in Figure 7. From Figure 8 it is clear how fast the observer estimates the states of the nonlinear function, external disturbances.

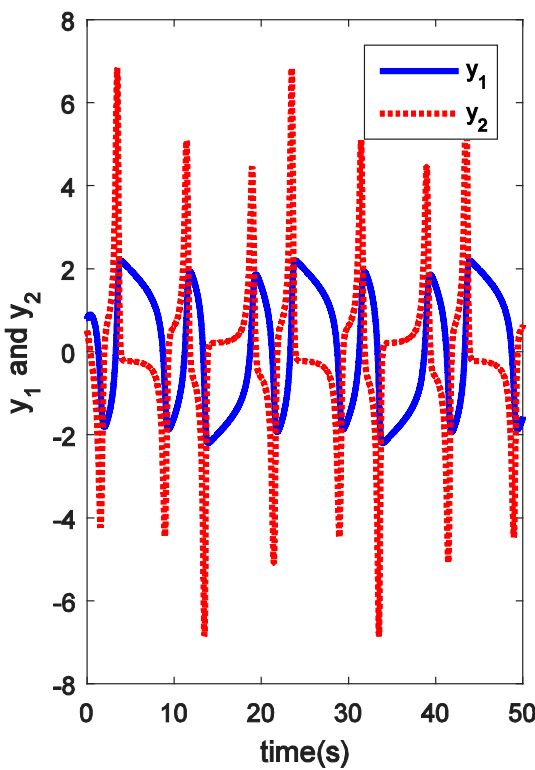

(a)

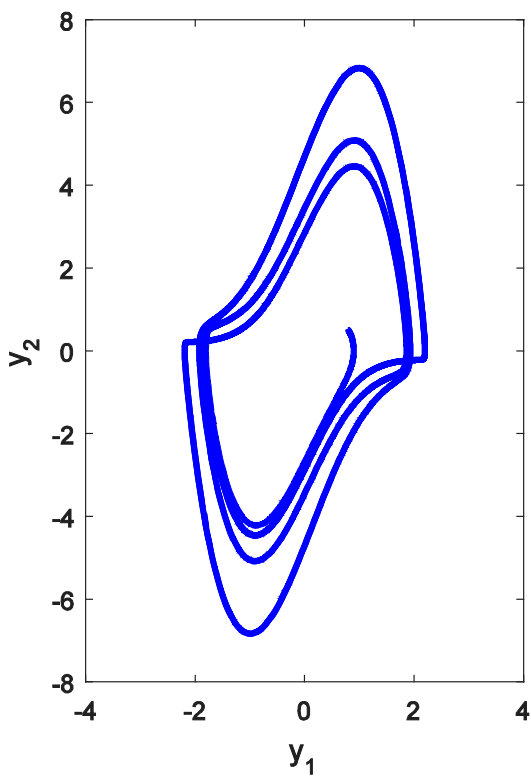

(b)

Figure 3. (a) The response of uncontrolled VdP system; (b) The phase plot.

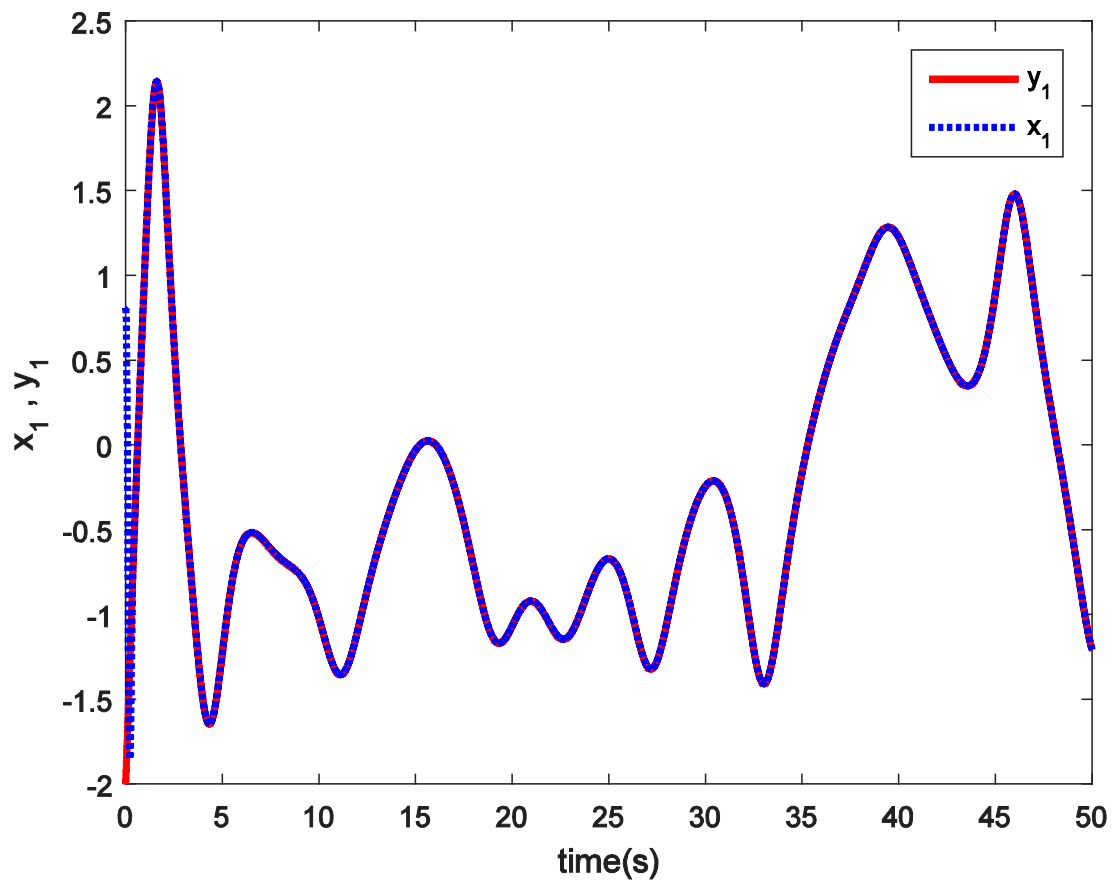

Figure 4. Synchronization of $y_{1}$ to $x_{1}$. 


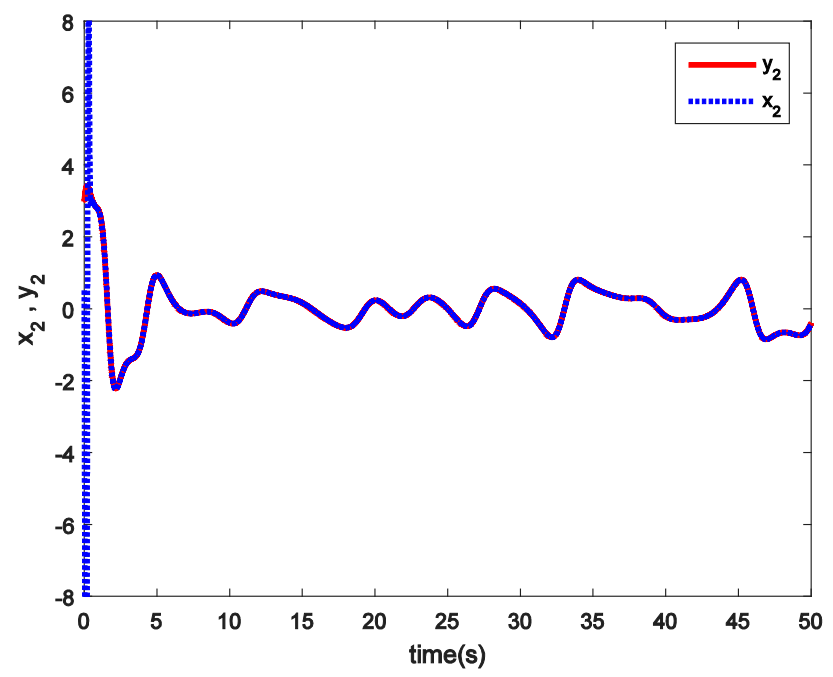

Figure 5. Synchronization of $y_{2}$ to $x_{2}$.
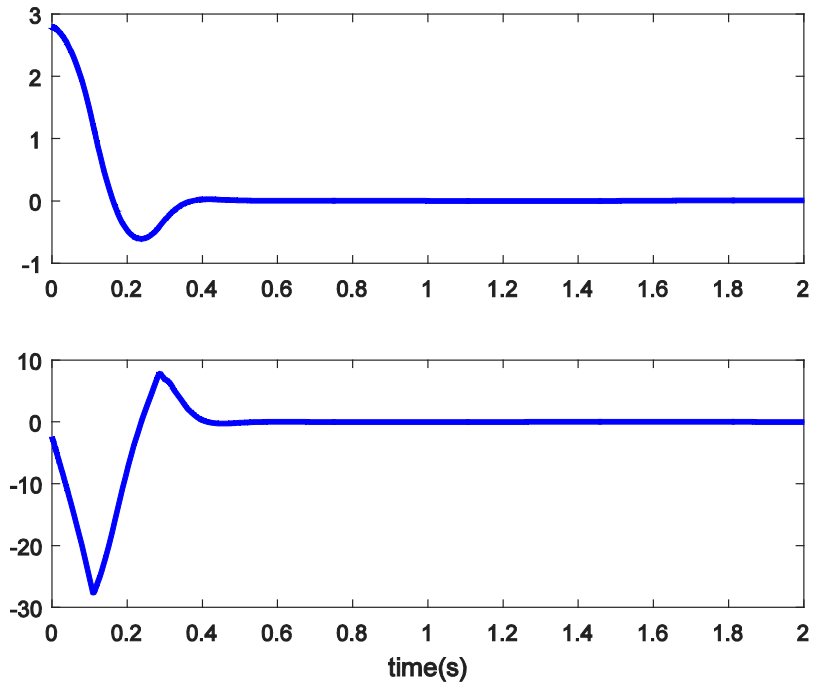

Figure 6. The errors as function of time.

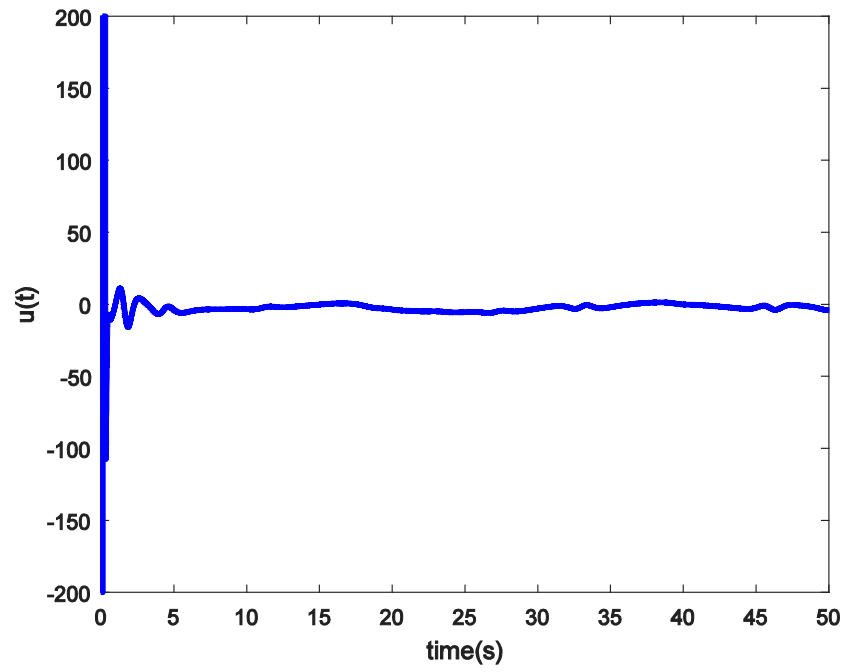

Figure 7. Control signal $u(t)$. 
(a)

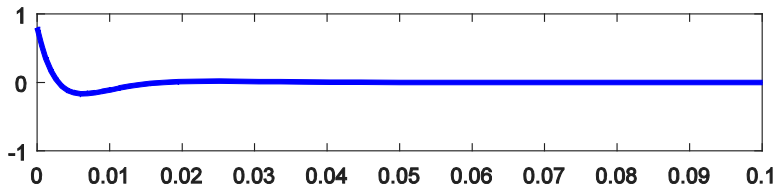

(b)

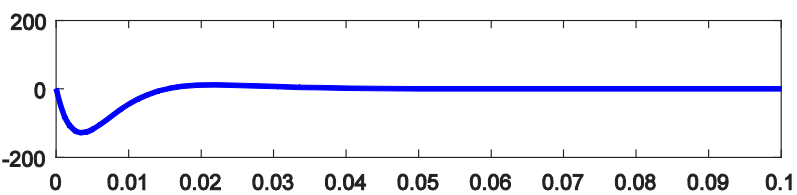

(c)

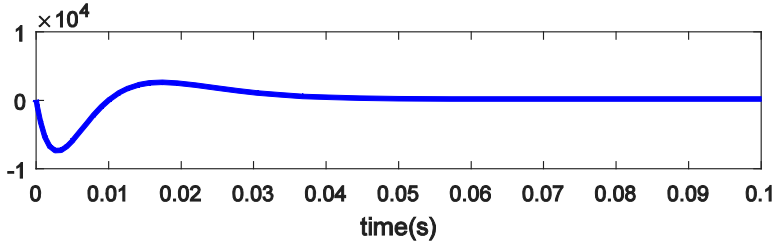

Figure 8. Estimation errors as a function of time (a) $e_{2}=\hat{y}_{1}-y_{1}$ (b) $e_{2}=\hat{y}_{2}-y_{1}$ and (c) $e_{3}=\hat{y}_{3}-\sigma$.

\section{Conclusion}

In this paper, the active disturbance rejection control methodology is used to synchronize two different chaotic systems. The idea of the ADRC is the use of a feedback controller coupled with an extended observer. The performance of the controller was verified through the numerical simulation. The results verified the performance of the controller in providing the convergence of error in a very short time. From the simulation results, it is concluded that the suggested method can be effectively used to synchronize chaotic systems in the presence of disturbances and uncertainties.

\section{References}

[1] Park, J.H., Lee, S.M. and Kwon, O.M. (2007) Adaptive Synchronization of GenesioTesi Chaotic System via a Novel Feedback Control. Physics Letters A, 371, 263-270.

[2] Ahn, K. (2010) Generalized Passivity-Based Chaos Synchronization. Applied Mathematics and Mechanics, 31, 1009-1018. https://doi.org/10.1007/s10483-010-1336-6

[3] Rodriguez, A., De Leon, J. and Fridman, L. (2007) Generalized Synchronization in Reduced-Order by Quasi-Continuous High-Order Sliding-Mode Controllers. 46th IEEE Conference on Decision and Control, New Orleans, 12-14 December 2007, 3703-3708.

[4] Handa, H. and Sharma, B.B. (2016) Novel Adaptive Feedback Synchronization Scheme for a Class of Chaotic Systems with and without Parametric Uncertainty. Chaos, Solitons \& Fractals, 86, 50-63.

[5] Sharma, B. and Kar, I.N. (2011) Observer-Based Synchronization Scheme for a Class of Chaotic Systems Using Contraction Theory. Nonlinear Dynamics, 63, 429445. https://doi.org/10.1007/s11071-010-9813-4

[6] Chen, S., Hu, J., Wang, C. and Lü, J. (2004) Adaptive Synchronization of Uncertain Rössler Hyperchaotic System Based on Parameter Identification. Physics Letters A, 321, 50-55.

[7] Yassen, M. (2005) Chaos Synchronization between Two Different Chaotic Systems 
Using Active Control. Chaos, Solitons \& Fractals, 23, 131-140.

[8] Khanesar, M.A., Teshnehlab, M. and Kaynak, O. (2012) Control and Synchronization of Chaotic Systems Using a Novel Indirect Model Reference Fuzzy Controller. Soft Computing, 16, 1253-1265. https://doi.org/10.1007/s00500-012-0810-Z

[9] Zhang, Z., Lu, J., Gao, L. and Shao, H. (2013) Exponential Synchronization of Genesio-Tesi Chaotic Systems with Partially Known Uncertainties and Completely Unknown Dead-Zone Nonlinearity. Journal of the Franklin Institute, 350, 347-357.

[10] Wang, G. (2010) Stabilization and Synchronization of Genesio-Tesi System via Single Variable Feedback Controller. Physics Letters A, 374, 2831-2834.

[11] Yadav, V.K., Mishra, P.K., Srivastava, M., Agrawal, S.K. and Mishra, V. (2016) Synchronization between Non-Autonomous Hyperchaotic Systems with Uncertainties Using Active Control Method. Procedia Computer Science, 79, 963-970.

[12] Liu, Y. and Lee, S.M. (2016) Synchronization Criteria of Chaotic Lure Systems with Delayed Feedback PD Control. Neurocomputing, 189, 66-71.

[13] Rodríguez, A., De León, J. and Fridman, L. (2009) Synchronization in ReducedOrder of Chaotic Systems via Control Approaches Based on High-Order SlidingMode Observer. Chaos, Solitons \& Fractals, 42, 3219-3233.

[14] Yahyazadeh, M., Ranjbar Noei, A. and Ghaderi, R. (2011) Synchronization of Chaotic Systems with Known and Unknown Parameters Using a Modified Active Sliding Mode Control. ISA Transactions, 50, 262-267.

[15] Bai, E.-W. and Lonngren, K.E. (1997) Synchronization of Two Lorenz Systems Using Active Control. Chaos, Solitons \& Fractals, 8, 51-58.

[16] Lin, T.-C., Chen, M.-C. and Roopaei, M. (2011) Synchronization of Uncertain Chaotic Systems Based on Adaptive Type-2 Fuzzy Sliding Mode Control. Engineering Applications of Artificial Intelligence, 24, 39-49.

[17] Lin, T.-C. and Kuo, C.-H. (2011) Synchronization of Uncertain Fractional Order Chaotic Systems: Adaptive Fuzzy Approach. ISA Transactions, 50, 548-556.

[18] Feng, J., He, L., Xu, C., Austin, F. and Wu, G. (2010) Synchronizing the Noise-Perturbed Genesio Chaotic System by Sliding Mode Control. Communications in Nonlinear Science and Numerical Simulation, 15, 2546-2551.

[19] Gao, Z. (2006) Active Disturbance Rejection Control: A Paradigm Shift in Feedback Control System Design. 2006 American Control Conference, Minneapolis, 14-16 June 2006.

[20] Han, J. (2009) From PID to Active Disturbance Rejection Control. IEEE Transactions on Industrial Electronics, 56, 900-906. https://doi.org/10.1109/TIE.2008.2011621

[21] Yoo, D., Yau, S.S.-T. and Gao, Z. (2007) Optimal Fast Tracking Observer Bandwidth of the Linear Extended State Observer. International Journal of Control, 80, 102-111. https://doi.org/10.1080/00207170600936555 
Submit or recommend next manuscript to SCIRP and we will provide best service for you:

Accepting pre-submission inquiries through Email, Facebook, LinkedIn, Twitter, etc. A wide selection of journals (inclusive of 9 subjects, more than 200 journals)

Providing 24-hour high-quality service

User-friendly online submission system

Fair and swift peer-review system

Efficient typesetting and proofreading procedure

Display of the result of downloads and visits, as well as the number of cited articles Maximum dissemination of your research work

Submit your manuscript at: http://papersubmission.scirp.org/

Or contact ica@scirp.org 